\title{
Investigation of the relationship between sample size and risk factors for complex diseases based on a simulation study
}

\author{
Yasuyuki Tomita ${ }^{1,2}$, Masahiro Nakatochi ${ }^{1}$, Hiroyuki Asano ${ }^{3}$, Hideo Izawa ${ }^{3}$, \\ Mitsuhiro Yokota ${ }^{4}$ and Hiroyuki Honda ${ }^{1,5^{*}}$ \\ ${ }^{I}$ Department of Biotechnology, School of Engineering, Nagoya University, Furo-cho, Chikusa-ku, \\ Nagoya 464-8603, Japan \\ ${ }^{2}$ Research Fellow of the Japanese Society for the Promotion of Science (JSPS), 8 Ichiban-cho, \\ Chiyoda-ku, Tokyo 102-8472, Japan \\ ${ }^{3}$ Department of Cardiology, Nagoya University Graduate School of Medicine, 65 Tsurumai-cho, \\ Showa-ku, Nagoya 466-8550, Japan \\ ${ }^{4}$ Department of Genome Science, School of Dentistry, Aichi-Gakuin University, 2-11 Suemori-dori, \\ Chikusa-ku, Nagoya 464-8651, Japan \\ ${ }^{5}$ MEXT Innovative Research Center for Preventive Medical Engineering, Nagoya University, Furo-cho, \\ Chikusa-ku, Nagoya 464-8603, Japan \\ *E-mail: honda@nubio.nagoya-u.ac.jp
}

(Received April 7, 2007; accepted September 25, 2007; published online October 22, 2007)

\begin{abstract}
The correlation between major disease factors and sample size remains an important question in clinical investigations. A small sample size results in the selection of falsely significant risk factors that are not derived from population data. This problem is more serious in studies on multifactorial diseases based on polymorphisms and environmental factors because these studies require combination analysis.

In the present study, we defined threshold lines to identify risk factors comprising complex interactions based on sample size. These threshold lines were constructed by a simulation study based on a resampling method that comprised a large data set (1441 case subjects with myocardial infarction and 979 control subjects). Finally, we demonstrated that these threshold lines could be used to identify risk factors for different data sets. In conclusion, these threshold lines enable us to design an association study of multifactorial diseases based on combination analysis.
\end{abstract}

Key Words: Complex Diseases, Single Nucleotide Polymorphism, Sample Size

Area of Interest: Disease Mechanism and Control Model 


\section{Introduction}

The development of multifactorial diseases and the effect of treatment measures for them may merely have a modest uniform probability across individuals. Alternatively, some individuals might be highly responsive to the treatment, while others, highly resistant. In our previous study, we reported a method for predicting and classifying the risk factors for each individual [1][2][3][4]. In the case of myocardial infarction (MI), which is a multifactorial disease, the genetic factors responsible for susceptibility were observed to differ among patients based on environmental factors and the influence of other susceptibility genes.

A small sample size is one of the key features of expensive clinical investigations that have limited resources with regard to funding and time. To date, criteria have rarely been proposed for detecting the interaction between genes and environmental factors in multifactorial diseases with regard to sample size. Such a type I error is easily augmented when a large number of markers combined with a small sample size are tested simultaneously and independently [5]. Further, a small sample size also results in the selection of falsely significant risk factors that have not been derived from population data. This is especially true for association studies of multifactorial diseases, particularly lifestyle-related diseases such as cancer, diabetes mellitus, and MI that require combination analysis. Therefore, to design clinical investigations, it is important to study the correlation between major disease factors and sample size.

In the present study, by using a resampling method, we determined criteria for the selection of risk factors in the case of differences in sample proportions between case and control subjects. The resampling method [6][7] enables accurate error estimation for microarray data analysis by using a simple procedure that only requires computing time [8][9]. We applied this method to an association study on a combination of polymorphisms and environmental factors in order to control the sampling bias which represents different characteristics from population data and is mainly caused by a smaller sample size and analyzing a combination of several factors. Hence, it is necessary to adjust the criteria depending on the sample size. In this simulation study, we resampled subjects from studies with various sample sizes and investigated the characteristics of a statistical power of association test in a combination analysis. We used 2 types of datasets to construct and validate threshold lines. We demonstrated that such ideal lines obtained from the simulation study using large-scale data sets are powerful tools for identifying risk factors.

\section{Materials and Methods}

\subsection{Subjects and data on polymorphisms and environmental factors}

The aim of this study is to define the difference $\left(P_{1}-P_{0}\right)$ between 2 proportions, namely, that of exposed case $\left(P_{1}\right)$ and exposed control subjects $\left(P_{0}\right)$, and to construct threshold lines in a combination analysis for selecting risk factors based on sample size via a simulation study. $P_{1}$ represent the proportion of the case subjects having certain alleles or genotypes and environmental factors to all case subjects, while $P_{0}$ is the proportion of the control subjects having the same factors to all control subjects. We used the resampling method [6][7] to control the sampling bias, and we examined the regulated criteria for $P_{1}-P_{0}$ with regard to sample size by using the large data set described below.

This simulation study comprised 3514 Japanese subjects; 1975 of these (1441 males and 534 females) had MI, while 1539 (979 males and 560 females) did not exhibit any MI symptoms (Table 1). Further, 22 polymorphisms for males and 20 polymorphisms for females and 6 environmental 
factors were included. The environmental factors were converted into binary data by using a clinical protocol [10]. Sex-based differences in the association between genetic polymorphisms and the risk of MI can be attributed, at least in part, to the difference in the levels of estrogen or other hormones between males and females [11]. Therefore, we analyzed the male and female subjects separately. There was no missing data regarding polymorphisms and environmental factors for any of the subjects. The combination analysis involved up to 3 factors: (1) 1 polymorphism and 1 environmental factor, (2) a combination of 1 polymorphism and 1 environmental factor and a combination of 2 polymorphisms, and (3) a combination of 2 polymorphisms and 1 environmental factor and a combination of 3 polymorphisms. Evidence that risk factors identified using modeling data were useful in predicting disease development with blinded data for validation analyses was obtained from a 10 -fold cross-validation analysis reported previously [3]. In addition, since the data sets, particularly that of the male subjects, were large, we considered them as hypothetical population data and used them in the resampling method described below.

Table 1. Number of subjects, polymorphisms, and environmental factors examined in the present study.

There was no missing data regarding polymorphisms and environmental factors for any of the subjects.

\begin{tabular}{|c|c|c|c|}
\hline \multicolumn{2}{|c|}{$\begin{array}{c}\text { males } \\
2420 \\
\end{array}$} & \multicolumn{2}{|c|}{$\begin{array}{c}\text { females } \\
1094 \\
\end{array}$} \\
\hline case & control & case & control \\
\hline 1441 & 979 & 534 & 560 \\
\hline polymorphisms & environmental factors & polymorphisms & environmental factors \\
\hline 22 (16 genes) & 6 & 20 (16 genes) & 6 \\
\hline
\end{tabular}

We generated a rule table in the combination analysis; each square matrix in Figure 1 represents 1 rule. For example, in rule 1 of Figure 1, subjects with genotype AA of SNP A, the B allele of SNP B, and a negative of the environmental factor (e.g., a positive means that subjects are diabetes mellitus and negative means that subjects are not.), are considered to represent one of the rules for using the $2 \mathrm{SNP}$ and 1 environmental factor combination. In each combination, exhaustive combinations of dominant and recessive genotypes were considered, for example, $2^{g}$ dominant and recessive combinations for a combination of $g$ SNPs. Thus, a combination of $g$ SNPs and 1 environmental factor comprised $2^{g}$ (genotype combinations) $\times 2^{g+1}$ (rules in a genotype combination) possibilities. Statistical tests were performed for each rule as described below. 


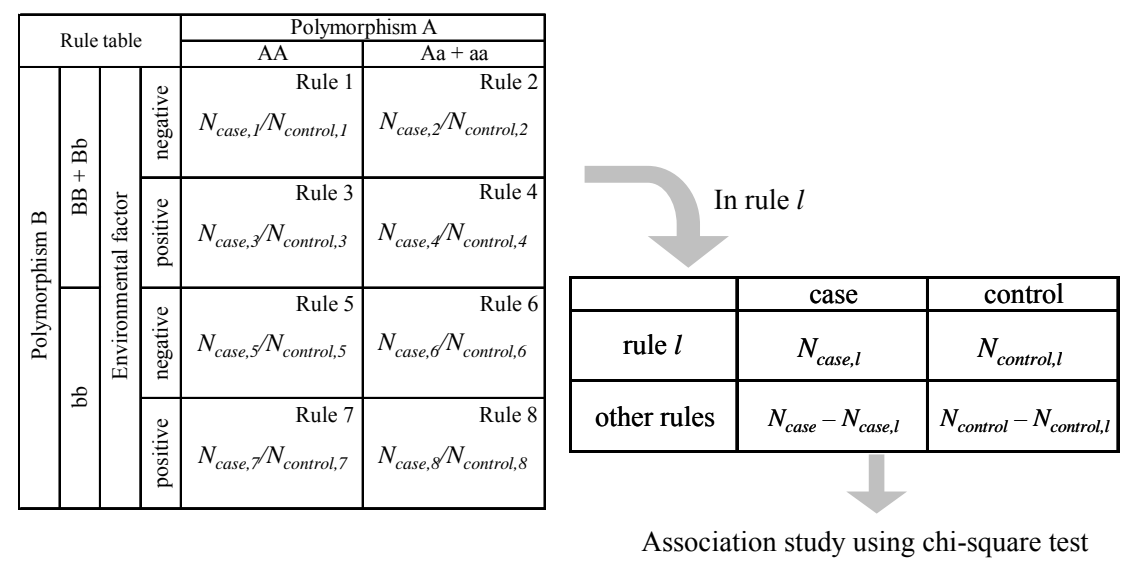

Figure 1. Rule table generated using a combination of 2 polymorphisms and 1 environmental factor.

$N_{\text {case, },}$ and $N_{\text {control, } l}$ represent the number of case and control subjects, respectively, belonging to rule $l$. $N_{\text {case }}$ and $N_{\text {control }}$ represent the total number of case and control subjects assessed in the combination analysis.

\subsection{Association test}

For the association analysis of a combination of factors, we performed the chi-square test by using the $2 \times 2$ table. In other words, the proportion of the case subjects $\left(\hat{P}_{1}=N_{\text {case }, l} / N_{\text {case }}\right)$ in the rule on certain alleles or genotypes and environmental factors (rule $l$ in Figure 1) was compared with that of the control subjects $\left(\hat{P}_{0}=N_{\text {control, } l} / N_{\text {control }}\right)$ in the rule. $N_{\text {case }}$ and $N_{\text {control }}$ represent the total number of case and control subjects, respectively, analyzed in the combination analysis. $N_{\text {case, } l}$ and $N_{\text {control, }}$ represent the number of case and control subjects, respectively, that belonged to rule $l$. Under the null hypothesis $\mathrm{H}_{0}$ of no association between the rule and the disease, the test statistic $\mathrm{Z}$ value is as follows:

$$
Z=\frac{\hat{P}_{1}-\hat{P}_{0}}{\sqrt{\hat{P}(1-\hat{P})\left(\frac{1}{N_{\text {case }}}+\frac{1}{N_{\text {control }}}\right)}}
$$

$\hat{P}_{1}$ and $\hat{P}_{0}$ represent the sample proportions of the case and control subjects in the rule. $\hat{P}$ represents the combined proportion of the case and control subjects in the rule $\left(\hat{P}=\left(N_{\text {case }, l}+\right.\right.$ $\left.\left.N_{\text {control }, l}\right) /\left(N_{\text {case }}+N_{\text {control }}\right)\right)$. Additionally, because we considered risk factors in the present study, the association study was performed for the rules in which $N_{\text {case, }} \times\left(N_{\text {control }}-N_{\text {control, },}\right)-N_{\text {control, } l} \times$ $\left(N_{\text {case }}-N_{\text {case }, l}\right)$ was greater than $0\left(\hat{P}_{1}-\hat{P}_{0}>0\right)$.

Further, under the alternative hypothesis $\mathrm{H}_{1}$ of the association between the rule and the disease, $P_{1}$ and $P_{0}$ are also obtained as shown above $\left(P_{1}=N_{\text {case, },} / N_{\text {case }}, P_{0}=N_{\text {control, },} / N_{\text {control }}\right)$. The following formula should be satisfied to obtain a power of $1-\beta$ for a significance level of $\alpha$ [12].

$$
Z_{1-\beta}=\frac{Z_{\alpha} \sqrt{(1+S) P(1-P)}-\left(P_{1}-P_{0}\right) \sqrt{N_{\text {case }}}}{\sqrt{S P_{0}\left(1-P_{0}\right)+P_{1}\left(1-P_{1}\right)}}
$$

$S$ represents the proportion of the case subjects to the total number of control subjects $(S=$ $\left.N_{\text {case }} / N_{\text {control }}\right)$ that were assessed in the combination analysis. $P$ represents the combined proportion of the case and control subjects in the rule $\left(P=\left(N_{\text {case }, l}+N_{\text {control, }, l}\right) /\left(N_{\text {case }}+N_{\text {control }}\right)\right)$. In 
this study, it is assumed that $Z_{\alpha}=1.64$ (corresponds to $\alpha=0.05$ ).

\subsection{Resampling method}

In order to define the difference in sample proportions $\left(P_{1}-P_{0}\right)$ and to construct threshold lines for the combination analysis, a simulation study based on the resampling methods was performed as has been described below and outlined in Figure 2. This iterative process is assumed to be the sampling step in clinical investigations. We paid special attention to identifying risk factors based on sample size without a sampling bias. The 2420 male subjects (1441 cases and 979 controls) were used to obtain the threshold lines, and the 1094 female subjects (534 cases and 560 controls) were used to demonstrate the validity of these lines. Prior to the simulation study, we investigated the relationship between specificity and sensitivity (equations 3 and 4, respectively) and that between specificity and $P_{1}-P_{0}$, as shown in Figure 3 and Figure 4, respectively (rules wherein $P_{1}-$ $P_{0}$ was greater than 0$)$.

$$
\begin{aligned}
& \text { specificity }=\frac{N_{\text {control }}-N_{\text {control }, l}}{N_{\text {control }}} \\
& \text { sensitivity }=\frac{N_{\text {case }, l}}{N_{\text {case }}}
\end{aligned}
$$

The specificity and sensitivity were strongly correlated; for MI, the higher the specificity was, the lower the sensitivity was and vice versa, as reported in a previous study [3]. In other words, in multifactorial diseases such as MI, there is no conclusive risk factor that exhibits both high specificity and sensitivity. It is true that their environmental factors and susceptible genetic factors are different between the male and the female subjects [10], but the complexity of potential environmental factors and the contribution of the risk factors to the disease might be similar between them as shown in Figure 3. In addition, the $P_{1}-P_{0}$ value of the rule comprising potential risk factors of MI $(P$ value $<0.05$ and power $>0.8)$ was observed to vary depending on the specificity of the rule (Figure 4). Hence, we investigated the threshold line with respect to each specificity condition, as shown in Figure 5.

As described in Figure 2, we used the resampling method to construct threshold lines in order to control the sampling bias. This random resampling was performed without replacement. For random number generation in the resampling method, we implemented the Mersenne Twister algorithm [13]. The software program was obtained from the http://www.emit.jp/ website. First, n subjects of the 2420 male subjects were randomly selected, and all the rules $\left(P_{1}-P_{0}>0\right)$ were assessed via an association test (chi-square test using the $2 \times 2$ table). Prior to the association test, all the rules were classified under the specificity conditions based on the values that were calculated using the 2420 subjects. When the $P$ value of the rule was less than 0.05 , the power was calculated by using equation 2 . The resampling and association tests were iterated 1000 times in each rule. Next, we defined $95 \%$ quantile of power with a lower confidence level of $95 \%$ in each rule, provided all the $P$ values obtained for 1000 iterations were less than 0.05 . Thus, an error rate of within 5\% was accepted for the detection of risk factors. The quantile is often used to evaluate the statistical significance of the classifier. For example, $25 \%$ and $75 \%$ quantile error rates obtained by a random permutation test were used for assessing the statistical significance of the classification performance for a given dataset size and estimating the dataset size requirements for classifying DNA microarray data [14]. Finally, the rule for which the 95\% quantile value was greater than 0.8 was regarded as a risk factor for the sample size $n$, that is, the $5 \%$ error allowance mentioned above enabled the identification of the rule for which the $95 \%$ quantile value was the 
closest to 0.8 . The $P_{1}-P_{0}$ (calculated using 2420 subjects) of the rule for which the $95 \%$ quantile value was closest to 0.8 (between 0.79 and 0.82 ) was plotted as shown in Figure 5 . Thus, we examined the relationship between $P_{1}-P_{0}$ and sample size $\mathrm{n}$. Further, the sample size $\mathrm{n}$ was decreased by 100 over a range of 2400 to 1000 . If there was no rule for which the $95 \%$ quantile value was between 0.79 and 0.82 , the plot could not be obtained for the sample size $\mathrm{n}$ (deficiency of a plot in Figure 5).

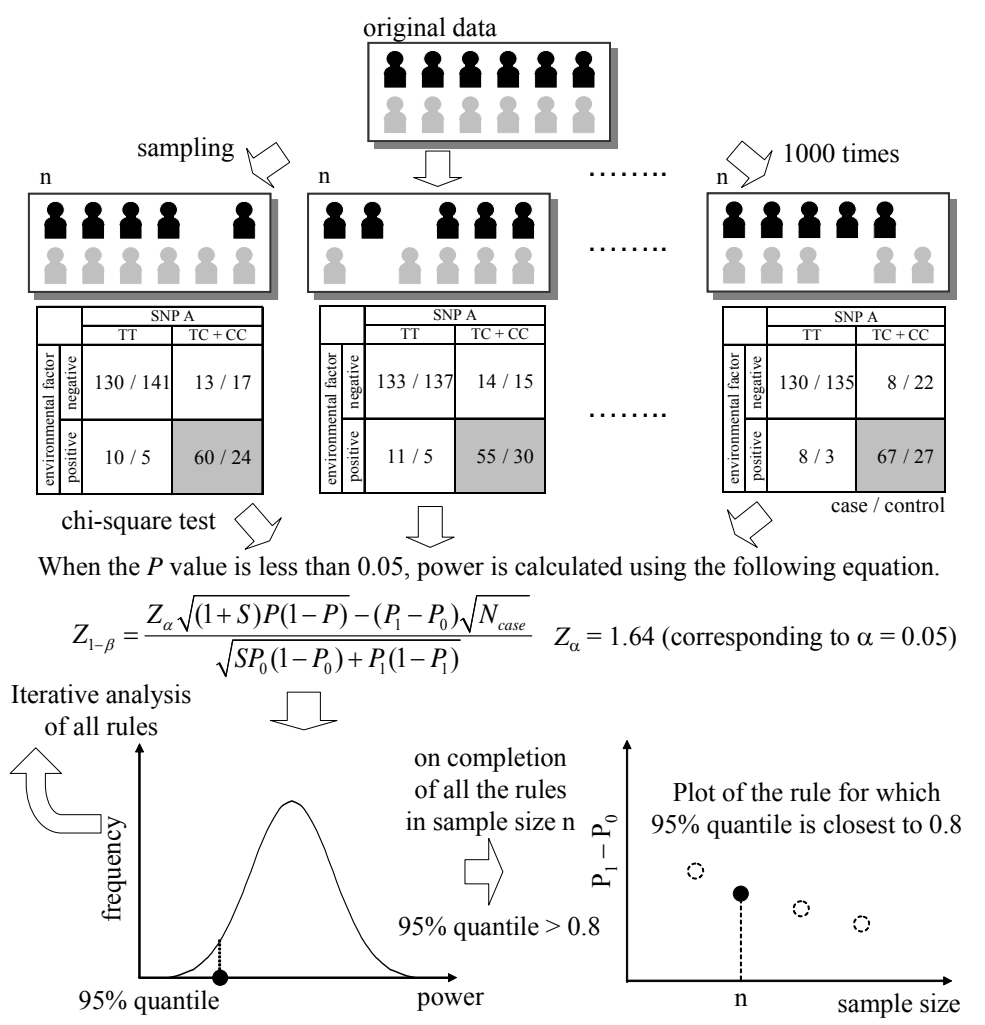

Figure 2. Summary of the resampling method to define threshold lines in order to control sampling bias in the combination analysis.

The line represents the threshold difference between 2 proportions, namely, that of the case $\left(P_{1}\right)$ and control $\left(P_{0}\right)$ subjects at the candidate rule. First, $\mathrm{n}$ of the 2420 male subjects were randomly selected, and all the rules with $P_{1}-P_{0}>0$ (exhaustive combinations of dominant and recessive genotypes for each combination analysis) were assessed by the chi-square test using the $2 \times 2$ table. The power was calculated using equation 2 when the $P$ value in the rule was less than 0.05 . The resampling and association tests were iterated 1000 times in each rule. Next, we defined the $95 \%$ quantile of power with a lower confidence level of $95 \%$ in each rule, provided all the $P$ values obtained for 1000 iterations were less than 0.05 . Finally, the rule for which the $95 \%$ quantile value was greater than 0.8 was regarded as a risk factor in sample size n, and $P_{1}-P_{0}$ of the rule for which the $95 \%$ quantile value was closest to 0.8 (between 0.79 and 0.82 ) was plotted, as shown in this figure. Thus, we examined the relationship between $P_{1}-P_{0}$ and sample size $\mathrm{n}$. The sample size was decreased by 100 over a range of 2400 to 1000 . If there was no rule for which the $95 \%$ quantile value was between 0.79 and 0.82 , the plot could not be obtained for the sample size $n$ (deficiency of a plot). 

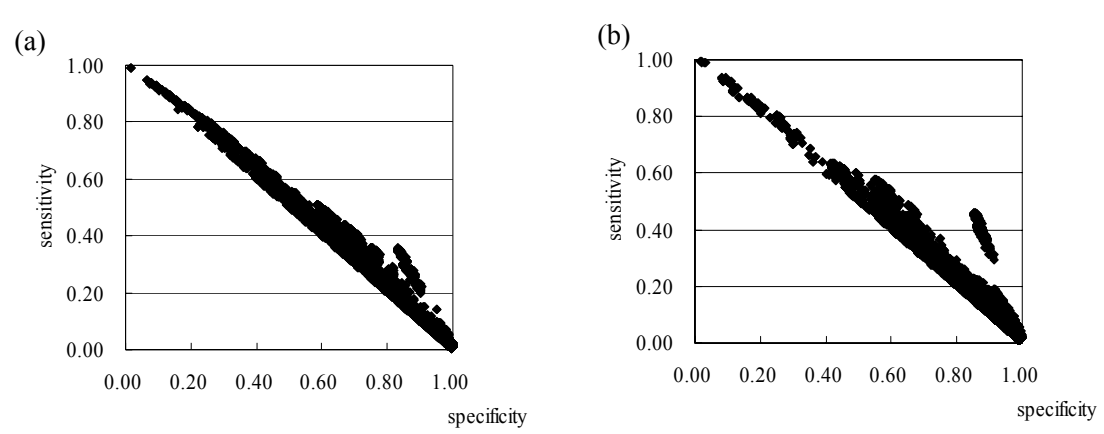

Figure 3. Relationship between specificity and sensitivity for MI in (a) male and (b) female subjects.

Each dot represents 1 rule for which the $N_{\text {case }, l} \times\left(N_{\text {control }}-N_{\text {control }, l}\right)-N_{\text {control }, l} \times\left(N_{\text {case }}-N_{\text {case }, l}\right)$ value listed in Figure 1 was greater than 0.

(a)

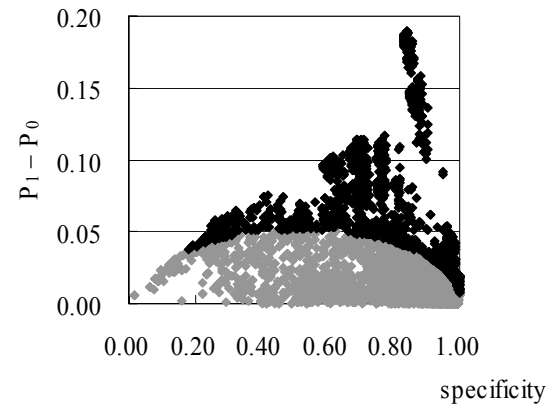

(b)

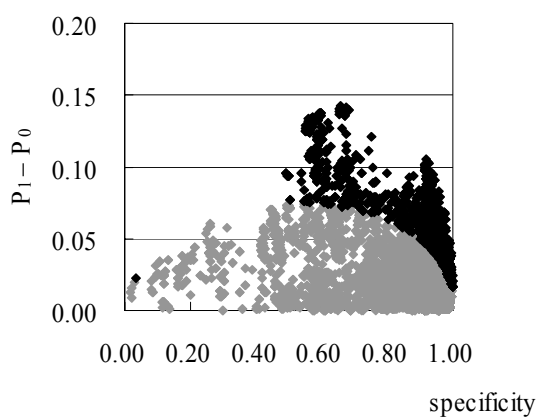

Figure 4. Relationship between specificity and $P_{1}-P_{0}$ in (a) male and (b) female subjects. Each dot represents 1 rule for which the $N_{\text {case }, l} \times\left(N_{\text {control }}-N_{\text {control, } l}\right)-N_{\text {control, }} \times\left(N_{\text {case }}-N_{\text {case }, l}\right)$ value listed in Figure 1 was greater than $0\left(P_{1}-P_{0}>0\right)$. The black dot in (a) and (b) represents the rule for which the $P$ value and power were less than 0.05 and greater than 0.8 , respectively.

\subsection{Evaluation of the threshold lines}

We used the 1094 female subjects (534 case and 560 control subjects) to demonstrate the validity of the threshold lines obtained using the resampling method with the male subjects. The procedure for this analysis comprised 3 steps. First, n subjects from among the 1094 subjects were sampled once, and the rules $\left(P_{1}-P_{0}>0\right)$ were classified under the specificity conditions based on their values. This process corresponds to the data sampling step in an association study. Second, the $P_{1}-P_{0}$ values of all the rules were calculated. The rules for which the $P_{1}-P_{0}$ values satisfied the threshold values $\left(P_{1}-P_{0}\right.$ greater than the value on the threshold line) were selected as rule group A. In contrast, the other rules for which the $P_{1}-P_{0}$ values did not satisfy the threshold values $\left(P_{1}-P_{0}\right.$ less than the value on the threshold line) were selected as rule group $\mathrm{B}$ in the comparative study. Finally, n subjects from among the 1094 subjects were randomly resampled 1000 times. Further, with respect to rules belonging to groups A and B, the number of times that each rule satisfied 2 conditions, namely, $P$ value (less than 0.05 ) calculated using the chi-square test and power $(1-\beta$, more than 0.8$)$ calculated using equation 2 was counted among the 1000 resamplings. This process corresponds to the validation analysis of the selected rules (group A rules). We performed this validation test using sample sizes of 900 and $700(n=900$ and 700$)$. 


\section{Results}

Initially, we investigated the relationship between specificity and sensitivity (equations 3 and 4, respectively) and that between specificity and $P_{1}-P_{0}$. As shown in Figure 3 and Figure 4, a high correlation was obtained between sensitivity and specificity, and the $P_{1}-P_{0}$ value of the rule comprising potential risk factors for MI $(P<0.05$ and power $>0.8)$ was observed to vary based on the specificity of the rule; therefore, we investigated the threshold line with respect to each specificity condition. As shown in Figure 5, the rules for which the 95\% quantile values of power were closest to 0.8 (between 0.79 and 0.82 ) were plotted for each sample size. The regression lines and coefficient of determination $\left(\mathrm{R}^{2}\right.$ value) obtained based on these plots are also shown in Figure 5. A clear linear relationship was obtained for each specificity condition. The smaller the sample size and the lower the specificity, the higher was the $P_{1}-P_{0}$ value required for a rule to be considered a significant risk factor. Threshold lines with a specificity of less than 0.5 were not obtained in the resampling method. The reason for these characteristics is believed to be that the rule with low specificity and high sensitivity comprised only a slightly higher number of case subjects than control subjects with relatively large samples. This is because in multifactorial diseases such as MI, there is no rule that comprises a considerably higher number of case subjects than control subjects in a large sample size.

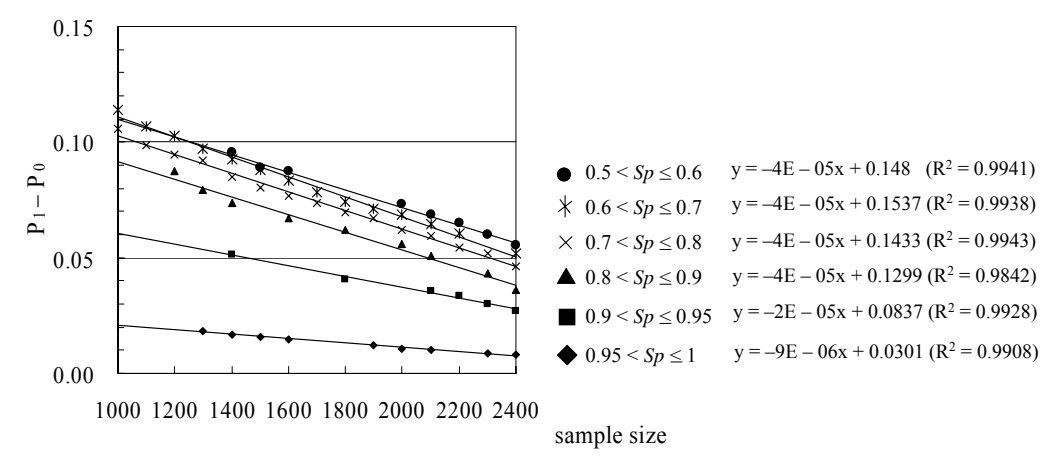

Figure 5. Threshold lines obtained in the combination analysis for identifying risk factors by using the difference between 2 proportions $\left(P_{1}-P_{0}\right)$ based on sample size.

$P_{1}$ and $P_{0}$ represent the sample proportions of the case and control subjects, respectively, in the rule of certain alleles or genotypes and environmental factors.

We used the 1094 female subjects (534 cases and 560 controls) to validate the threshold lines. Figure 6 shows the histogram of the times that satisfied both conditions in 1000 resamplings: a $P$ value of less than 0.05 and a power $(1-\beta)$ value of greater than 0.8 . Approximately $90 \%$ of the group A rules (757 and 477 rules in sample sizes 900 and 700, respectively) satisfied the required conditions between 900 to 1000 times for both sample sizes, as shown in Figure 6 (a) and (c). In contrast, as shown in Figure 6 (b) and (d), the frequency of the group B rules (5726 and 5993 rules in sample sizes 900 and 700, respectively) that satisfied the required conditions more than 500 times was considerably low as compared to that of the group A rules. In conclusion, since the rule that satisfied the threshold values in each sample size could be considered as a risk factor that is less affected by sampling bias, the threshold lines defined in the present study are useful for selecting risk factors based on sample size. 
(a)

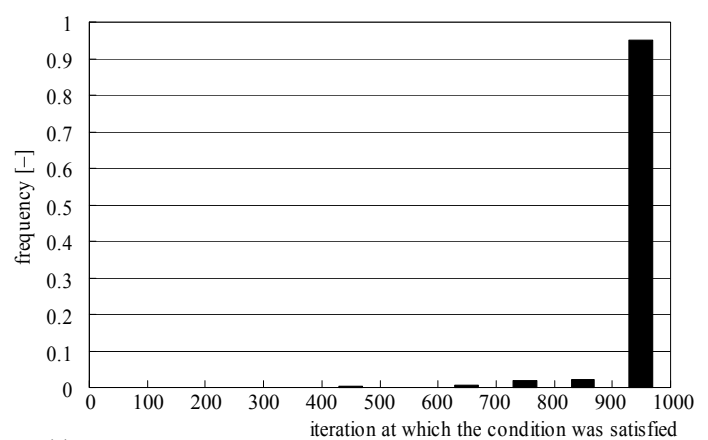

(1)

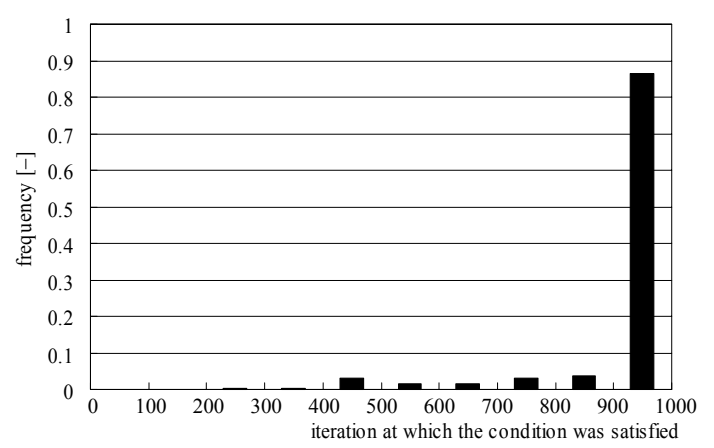

(b)

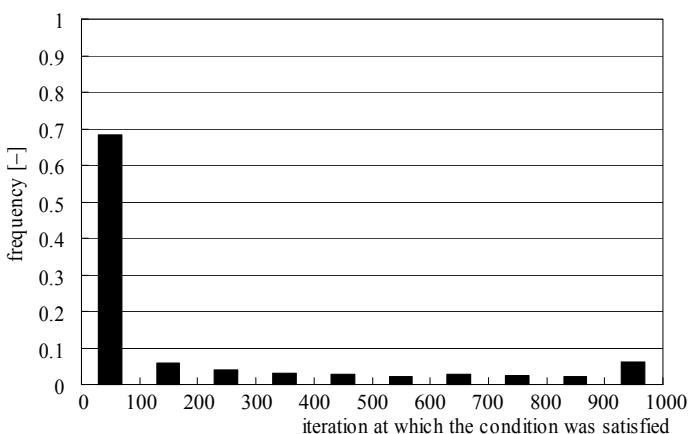

(d)

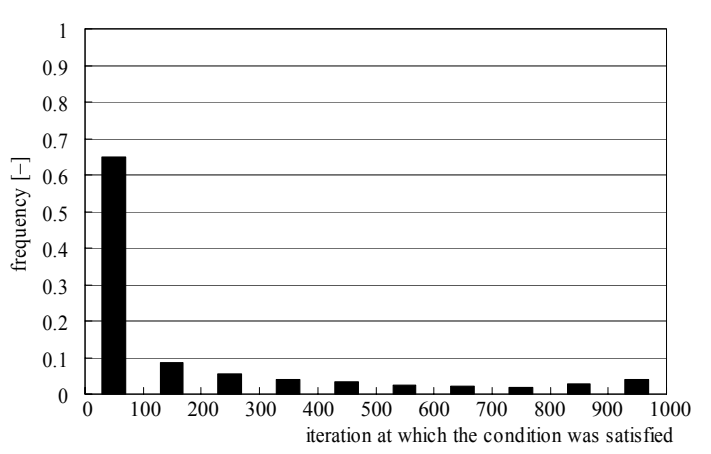

Figure 6. The evaluation of threshold lines defined using the resampling method in male subjects.

This figure shows the histograms of the iterations that satisfied both conditions, namely, $P$ value (less than 0.05$)$ calculated using the chi-square test and power $(1-\beta$, more than 0.8$)$ calculated using equation 2 in 1000 resamplings. The resampled sample sizes (n) and the rule group (A or B) mentioned in section 2.4 are as follows: (a) $(900, A),(b)(900, B),(c)(700, A)$, and (d) $(700, B)$.

\section{Discussion}

Interactions between genetic and environmental factors, including diet and lifestyle, contribute to cardiovascular diseases, cancers, and other major causes of mortality [15] that are considered to be multifactorial diseases. Such interactions alter or increase the risk of these diseases because they can modify either the transcription or translation levels directly or the protein products indirectly [16]. Further, it was considered that there are the risk factors relating to the development of a particular disease and all risk factors will be never required for development of the disease. Assuming that the hypothesis is acceptable, the actual risk factors for developing are believed to differ among individuals based on their environmental factors affecting susceptibility genes. In addition, some people develop the disease, while others resist it even if they have common risk factors. A reason for this appears to be the existence of a protective factor against the disease that may play a role in preventing its development [4][17]. Thus, for multifactorial diseases such as MI, there is no conclusive risk factor that many case subjects and few control subjects exhibit. This was evident in the relationship observed between sensitivity and specificity (Figure 3). Thus, the abovementioned multifactorial diseases appear to exhibit almost identical characteristics as those of MI with regard to sensitivity and specificity. 
In the present study, we defined the difference between 2 exposed sample proportions $\left(P_{1}-P_{0}\right)$ and constructed threshold lines to identify risk factors based on sample size. The smaller the sample size, the greater is the possibility that a rule will be estimated as falsely significant. The underlying reason is that the distribution of case and control subjects within a rule is considered to differ from that in a population due to the small sample size in the rule. This was evident in the threshold lines obtained (the smaller the sample size, the higher is the $P_{1}-P_{0}$ value required for the rule to be considered a significant risk factor). In addition, the lower the specificity, the higher is the $P_{1}-P_{0}$ value required for the rule to be considered a significant risk factor. Since these rules comprise only a slightly higher number of case subjects than control subjects with relatively large samples in multifactorial diseases, the rule should satisfy higher $P_{1}-P_{0}$ value compared with high-specificity and low-sensitivity rules if it is to be identified as a risk factor in small sample size. In contrast, high-specificity and low-sensitivity rules comprise relatively small sample sizes and considerably more case subjects than control subjects. This tendency is particularly prominent in rules for which the specificity lies between 0.95 and 1 . Further, the $P_{1}-P_{0}$ value required for the rule to be considered a significant risk factor appears to be low, and high-specificity rules appear to be less affected by sample size than low-specificity rules. Risk factors based on high-specificity rules can easily be detected using a relatively small sample size.

Finally, we demonstrated that the threshold lines obtained using the male subjects could be used to identify risk factors for MI in females. Similarly, in other lifestyle-related diseases, there is no conclusive risk factor that exhibits both high specificity and sensitivity, and their pattern might be the same as that shown in Figure 3. In conclusion, since these threshold lines were constructed in a combination analysis under various conditions with regard to sample size and specificity, they are expected to be useful and applicable for identifying risk factors in multifactorial diseases based on polymorphisms and environmental factors.

In case-control studies, these threshold lines might be used in 2 ways. First, using the obtained data set of sample size $\mathrm{n}$, a particular rule can be evaluated by calculating the $P_{1}-P_{0}$ and specificity values. If the $P_{1}-P_{0}$ value of the rule is higher than that on the threshold line, it is concluded that the rule is one of the potential risk factors with a significantly high $P_{1}-P_{0}$ value. Second, the required sample size n can be estimated using the $P_{1}-P_{0}$ value; this value can be used for classifying case and control subjects $(P<0.05$ and power $>0.8)$ under the condition less affected by sampling bias in the case-control study design. For this, it is necessary to define the predictable $P_{1}-P_{0}$ value of the case or control before sampling. For example, the following rule is considered: the difference between 2 exposed sample (116 case and 68 control subjects) proportions $\left(P_{1}-P_{0}\right)$ equals 0.0411 using 845 case and 707 control subjects $(P=0.0157$ and power $=0.807)$. In this case, the specificity is 0.904 . Although this rule satisfies both the conditions, namely, $P$ value less than 0.05 and power greater than 0.8 , required sample size is 2131 subjects for regarding this rule as a risk factor according to the threshold line. Indeed, this rule is considered to be a risk factor (not false positive but true positive) in the disease, but the rule with the difference between 2 exposed sample proportions has the possibility to be false positive using the sample size ( $n=1552)$ according to the simulation study. Thus, it is difficult to conclude the rule is true positive or false positive for the development by using the significant level and the power because population heterogeneity and sampling bias caused by complex background factors are inevitable, markedly increased, and difficult to identify them using small sample size in multifactorial diseases. Since the rule may be false positive for the sample size, we concluded that a large sample size is required for the validation of this rule without sampling bias. Thus, these threshold lines enable us to design an association study based on combination analysis. 


\section{References}

[1] Y. Tomita, S. Tomida, Y. Hasegawa, Y. Suzuki, T. Shirakawa, T. Kobayashi and H. Honda, Artificial neural network approach for selection of susceptible single nucleotide polymorphisms and construction of prediction model on childhood allergic asthma, $B M C$ Bioinformatics, 5, 120 (2004).

[2] H. Mutoh, N. Hamajima, K. Tajima, T. Kobayashi and H. Honda, Exhaustive exploring using Artificial Neural Network for identification of SNPs combination related to Helicobacter pylori infection susceptibility, CBIJ, 5, 15-26 (2005).

[3] Y. Tomita, H. Asano, H. Izawa, M. Yokota, T. Kobayashi and H. Honda, Classification method for predicting the development of myocardial infarction by using the interaction between genetic and environmental factors, IPSJ Digital Courier, 2, 691-709 (2006).

[4] Y. Tomita, M. Ikeda, H. Mutoh, T. Inada, N. Iwata, N. Ozaki and H. Honda, Association study between Apolipoprotein $L$ and schizophrenia by using exhaustive and rule-based combination analysis method for the identification of multilocus interactions. J. Biosci. Bioeng., 103, 303310 (2007).

[5] N. Risch, and K. Merikangas, The future of genetic studies of complex human diseases, Science, 273, 1516-1517 (1996).

[6] B. Efron, Bootstrap methods - Another look at the Jackknife, Ann. Statist., 7, 1-26 (1979).

[7] B. Efron, and R. Tibshirani, Improvements on cross-validation: The .632+ bootstrap method, $J$. Am. Stat. Assoc., 92, 548-560 (1997).

[8] C. Ambroise and G.J. McLachlan, Selection bias in gene extraction on the basis of microarray gene-expression data, Proc. Natl. Acad. Sci. U S A, 99, 6562-6566 (2002).

[9] W.J. Fu, R.J. Carroll and S. Wang, Estimating misclassification error with small samples via bootstrap cross-validation, Bioinformatics, 21, 1979-1986 (2005).

[10] Y. Yamada, H. Izawa, S. Ichihara, F. Takatsu, H. Ishihara, H. Hirayama, T. Sone, M. Tanaka and M. Yokota, Prediction of the risk of myocardial infarction from polymorphisms in candidate genes, N. Engl. J. Med., 347, 1916-1923 (2002).

[11] V. Guetta and R.O. Cannon, Cardiovascular effects of estrogen and lipid-lowering therapies in postmenopausal women, Circulation, 93, 1928-1937 (1996).

[12] A. Donner, Approach to sample size estimation in the design of clinical trials - a review, Stat. Med., 3, 199-214 (1984).

[13] M. Matsumoto and T. Nishimura, Mersenne twister: A 623-dimensionally equidistributed uniform pseudorandom number generator, ACM Trans. Model. Comput. Simul., 8, 3-30 (1998).

[14] S. Mukherjee, P. Tamayo, S. Rogers, R. Rifkin, A. Engle, C. Campbell, T.R. Golub and J.P. Mesirov, Estimating dataset size requirements for classifying DNA microarray data, J. Comput. Biol., 10, 119-142 (2003).

[15] W.C. Willett, Balancing life-style and genomics research for disease prevention, Science, 296, 695-698 (2002).

[16] T.A. Thornton-Wells, J.H. Moore and J.L. Haines, Genetics, statistics and human disease: analytical retooling for complexity, Trends Genet., 20, 640-647 (2004).

[17] J.H. Nadeau and E.J. Topol, The genetics of health, Nat. Genet., 38, 1095-1098 (2006). 